\title{
Sublingual Epidermoid Cyst Simulating a Plunging Ranula: 2 Rare Case Reports
}

\author{
Elshad Sadigov ${ }^{1}$, Yunus Afendiyev ${ }^{1}$, Chingiz Rahimov², Mahammad Davudov²,
} Jamal Musayev³, Sakhavat Quliyev², Bilal Aliyev²

\begin{abstract}
${ }^{1}$ Azerbaijan State Institute for Advanced Training of Doctors named after A. Aliyev ${ }^{2}$ Department of Maxillofacial surgery, Azerbaijan Medical University, Baku, Azerbaijan

${ }^{3}$ Department of Pathology, Azerbaijan Medical University, Baku, Azerbaijan
\end{abstract}

\begin{abstract}
*Corresponding Author: Mahammad Davudov, Department of Maxillofacial surgery, Azerbaijan Medical University, Baku, Azerbaijan, Tel: +99 450 6258883,Email: mahammad_davud@mail.ru
\end{abstract}

Received Date: January 31, 2018 Accepted Date: February 09, 2018 Published Date: February 15, 2018

Citation: Elshad Sadigov, Yunus Afendiyev, Chingiz Rahimov, Mahammad Davudov (2018). Sublingual Epidermoid Cyst Simulating a Plunging Ranula: 2 Rare Case Reports. POJ Dent Oral Care. 2(1):1-4.

\section{Abstract}

The incidence ranging of the dermoid and epidermoid cysts on the head and neck, from 1.6 to $7.0 \%$, and they represent less than $0.01 \%$ of all oral cavity cysts. Histologically, they can be further classified as epidermoid, dermoid or teratoid.

We analyzed two rare cases of large epidermoid cysts of the floor of the mouth, with sublingual as well as submental component resembling plunging ranula. Two female patients (age 15 and 16 y. o.), who's had sublingual and submental swellings of oral floor with breathing and swallowing difficulties. The suspected clinical diagnosis of the sublingual dermoid or epidermoid cysts were supported by the radiological finding after performing ultrasonography (USG), computed tomography (CT) and magnetic resonance imaging (MRI). On the radiological investigation the differentiation between epidermoid cysts, ranulas, cystic tumours, mucous extravasation phenomena and hygromas sometimes indistinguishable, leading in the determination of internal component differentiation and has pathognomonic signs to differentiate.

The lesions were surgically excised using an intraoral approach. Microscopic examinations revealed a dermoid cyst of the epidermoid type.

Keywords: Sublingual Epidermoid Cyst, Plunging Ranula, Ultrasonography, Computed Tomography, Magnetic Resonance Imaging

\section{Introduction}

Epidermoid and dermoid cysts in $7 \%$ of all cases can occur in the head and neck region. Congenital cysts of ectodermal origin are uncommon in the oral cavity (1.6\%), with epidermoid cysts rarely occurring there. They represent less than $0.01 \%$ of all oral cavity cysts [1]. The pathogenesis of the midline cysts of floor of mouth includes dysontogenetic, traumatic, and thyroglossal anomaly. Histologically, after Meyer these cystic lesions are categorized as epidermoid, dermoid, and teratoid [2]. These cysts can be defined as dermoid cysts when skin adnexa are found, epidermoid when the lining presents only epithelium, and teratoid cysts when other tissue such as muscle, cartilage, and bone are present [3]. The majority of oral dermoid and epidermoid cysts occur between the ages of 15-35 years, but they may also be present at the time of birth. In most cases, dermoid and epidermoid cysts are small during childhood and enlarge during adolescence. They may reach a large size, involving more than one anatomical area [4].

\section{Case 1}

A 15 year old girl presented with a midline painless swelling in the sublingual and submental regions of the neck. The swelling were becoming prominent on contraction of the mylohyoid muscle and was not moving with swallowing of the tongue. There were complaints of gradual increasing painless swelling of the floor of the mouth with difficulty in speech and swallowing for 1.5 years of duration. Dimension of the cyst was $6.7 \mathrm{~cm} \times 4.8 \mathrm{~cm}$, which was one of the large cysts described in the literature. In this case no history of trauma, operation, or prolonged fever were present. There was no evidence of cervical

Copyright:@ 2018 Mahammad Davudov, et al. This is an open-access article distributed under the terms of the Creative Commons Attribution License, which permits unrestricted use, distribution, and reproduction in any medium, provided the original author and source are credited. 
lymphadenopathy. On clinical examination, her vitals were within normal limits. Central nervous system, cardiovascular, chest and abdominal examinations revealed no abnormality. Laboratory investigations including hemogram and ESR were normal. Clinical diagnosis was plunging ranula.

\section{Case 2}

A 16 year old girl showed a swelling in oral cavity under the tongue and difficulties with chewing and swallowing of solid foods for the past 8 months. The mass was painless and the patient had no dyspnea, no previous history of surgery or trauma to the oral cavity or neck. The dimension of the cyst was $5.1 \mathrm{~cm}$ $x 3.9 \mathrm{~cm}$ and it was fluctuant, soft and non-mobile. There were no secondary changes in overlying mucosa, no inflammatory signs or lymphadenopathy associated with the lesion. Clinical examinations and laboratory investigations were in normal limits. Clinical diagnosis suggested possibility of plunging ranula and thyroglossal duct cyst.

USG of the neck was done on GE Logiq S7 Expert machine using $8-15 \mathrm{MHz}$ linear probe, $\mathrm{CT}$ of the neck was done on multislice Hitachi Presto, MRI of the neck was done on Philips Achieva 1,5 T. USG examination of both cases of the sublingual swelling showed cystic swelling with low echogenicity, internal particulate content with no internal vascularity on the color Doppler study (Figure 1). Both lesions revealed particulate internal contents with single floating echogenic lobules inside the cysts with no posterior acoustic shadow. In case 2 there were anechoic map-like areas situated bilaterally (Figure 2). On USG differential diagnosis of dermoid/epidermoid cyst and plunging ranula were kept.

In case 1 CT showed well-circumscribed cystic mass extending into the floor of the mouth (Figure 3 ). In case $1 \mathrm{MRI}$ of the head and neck revealed large midline lesion in the submental region extending into the sublingual space. STIR MR images in sagittal and coronal scans showed a high-intensity lesion extending inferiomedially to the genioglossus muscle and inferiorly to the mylohyoid muscle. Axial T1-weighted MRI showed a sharply circumscribed cystic mass with low signal intensity, axial T2weighted MRI showed lesion with high signal intensity (Figure 4). A differentiation of epidermoid cyst / plunging ranula was suggested. Diffusion weighted image (DWI) had showed hyperintense signal and diffusion restriction and apparent diffusion coefficient (ADC) sequences.

Figure 1: Case 1. Transverse (A) and longitudinal (B) USG images show the large homogeneous hypoechoic cystic lesion.

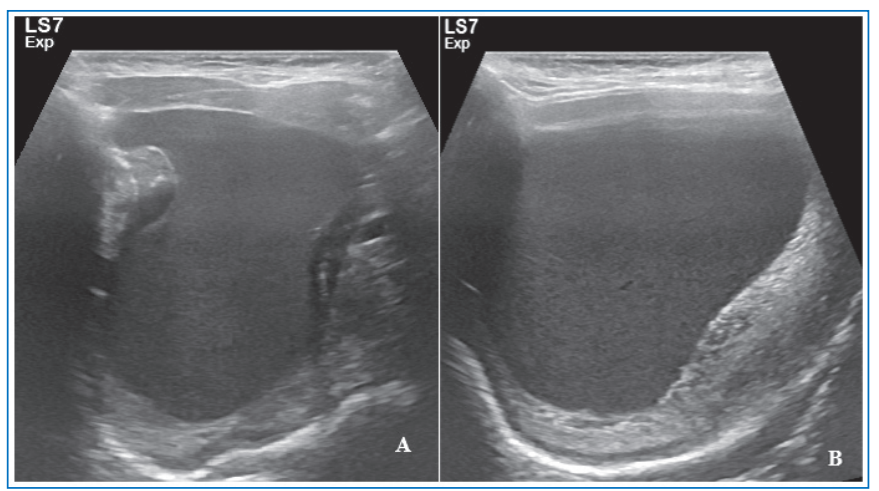

Figure 2: Case 2. Transverse (A) and longitudinal (B) USG images show the large hypoechoic cystic lesion with anechoic areas located bilaterally.

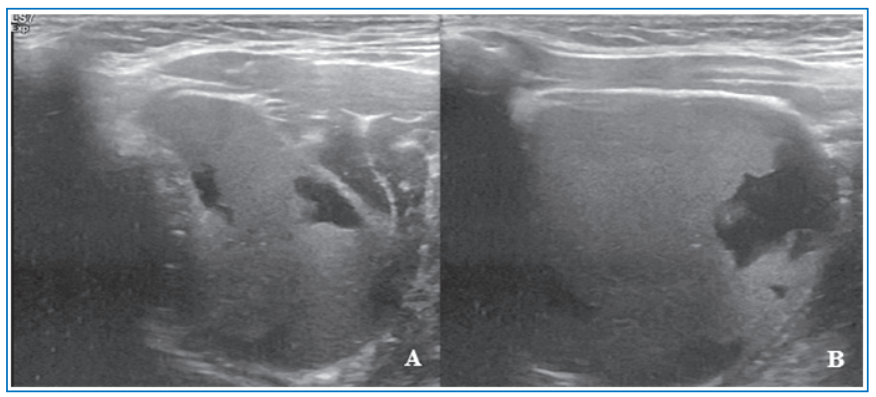

Figure 3: Case 1. Axial non-contrast CT image shows a well-defined, homogenous, hypo-attenuating cystic lesion located in midline between two genioglossus muscle.

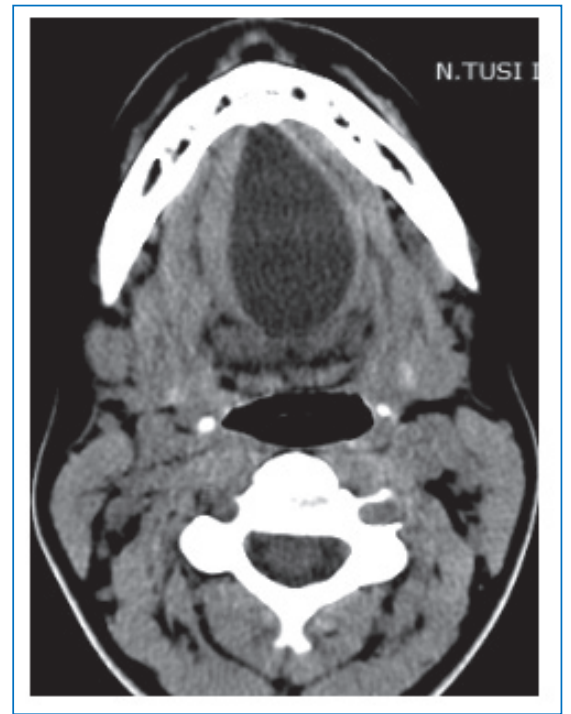

Figure 4: Case1. MRI of the neck in 15 year old girl without contrast. The nodular homogenous, well-defined lesion on STIR MR images (sagittal and coronal) and T2W image show high signal intensity (A, B, D), and on T1W image shows decreased signal intensity (C). This midline lesion occupied sublingual and submental spaces.

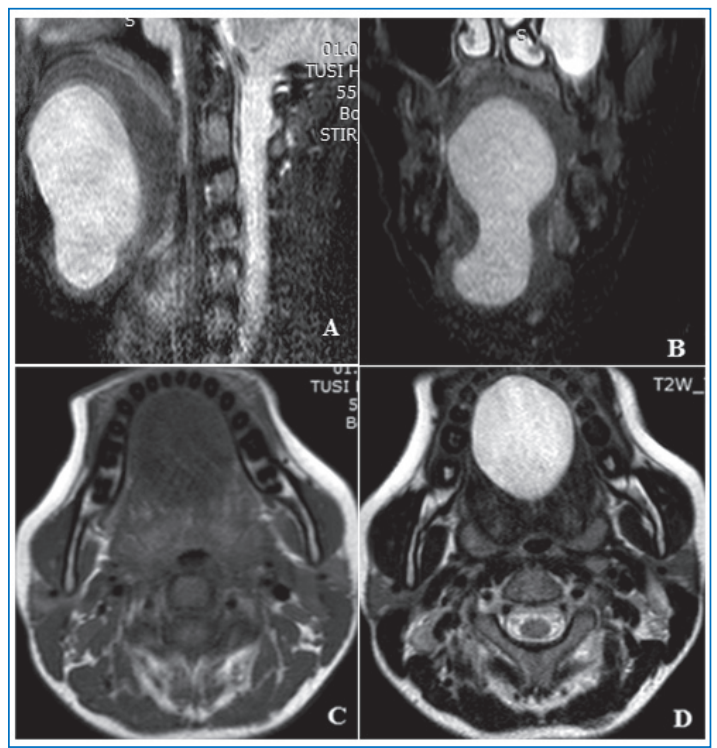

Citation: Elshad Sadigov, Yunus Afendiyev, Chingiz Rahimov, Mahammad Davudov (2018). Sublingual Epidermoid Cyst Simulating a Plunging Ranula: 2 Rare Case Reports. POJ Dent Oral Care. 2(1):1-4. 
In both of patients, we used intraoral approach as the cyst was easily accessible through this route and was easily excised. Both cystic lesions were enucleated by intraoral sublingual dissection with intact capsules, and the opening in the floor of the mouth was marsupialized (Figure 5). The sublingual cysts was below the floor of mouth mucosa. Histologically, internal surface of the cyst wall was covered by keratinized squamous epithelium in both cases (Figure 6). There were not any atypia and mitotic activity in the epithelial cells. After 6 and 12 months of follow up, in both cases, the cysts had not recurred.

Figure 5: Case 1. Intraoperative view of removing sublingual epidermoid cyst.

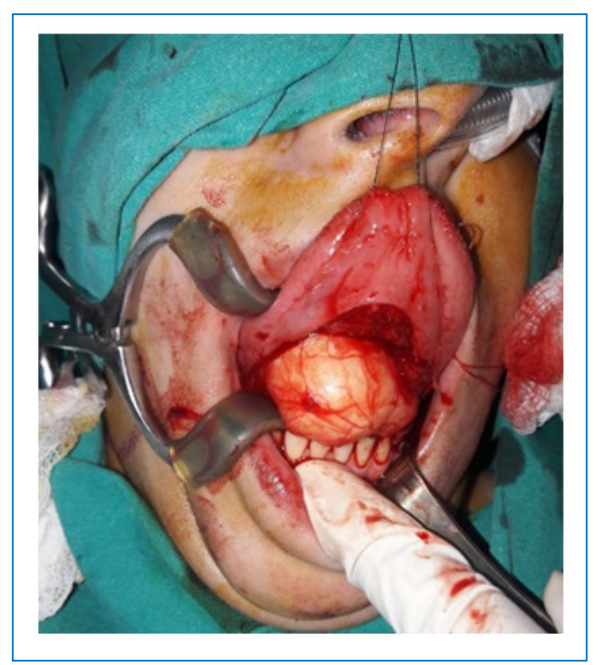

Figure 6: Case 1. Microscopic view of the cyst wall lined by keratinized squamous epithelium (hematoxylin\&eosin; $\times 100$ ).

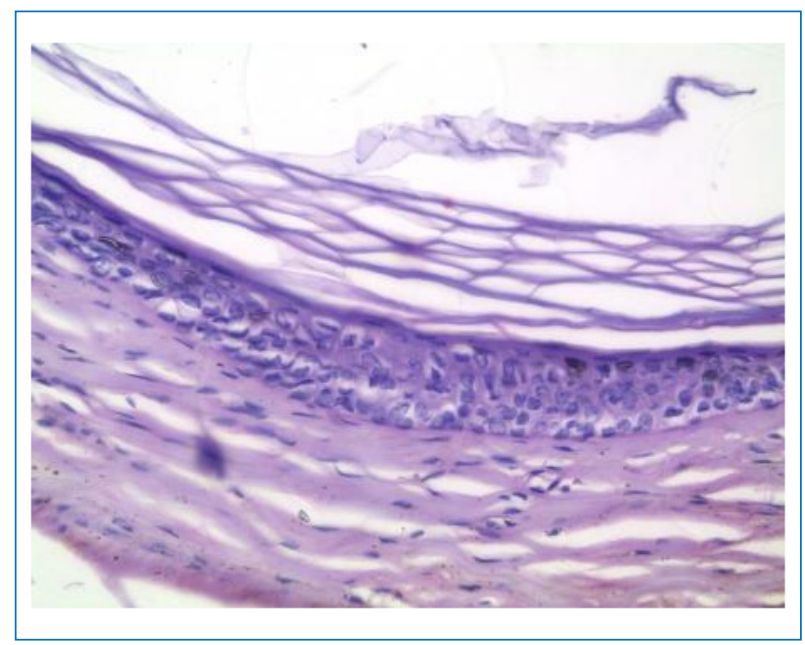

\section{Discussion}

Epidermoid cysts manifest earlier in life, with most lesions evident during infancy. The epidermoid cysts of the mouth are most frequently located on the median line of the mouth floor, most likely caused by the retention of the germinal epithelium during the growth of the mandible and hyoid branchial arches and are considered rare [3,5]. The congenital epidermoid cysts develop due to entrapment of ectodermal element between the first and second branchial arches during the 3rd and 4th weeks of intrauterine life [5]. These lesions typically manifest during the second or third decade of life, however may present since birth with equal frequency of occurrence in both genders.

Epidermoid cyst may extend laterally and downward and can attain a large size before presentation $[1,4,6]$. The plain radiographs and orthopantomogram are not of much help, the diagnosis is mainly made on high resolution ultrasound (HRUS), CT and MRI indicating the cystic nature of the tumor, its size and anatomical relations [7-10]. On imaging studies, epidermoid cysts will present as thin-walled, well-defined cystic lesions in the submandibular, sublingual or submental spaces. CT scan and MRI allow more precise localization of the lesion in relationship to mylohyoid and geniohyoid muscles, and enable the surgeon to choose the most appropriate surgical approach, especially for very large- and medium-sized lesions $[8,11]$. On USG epidermoid cysts usually seen as anechoic masses with posterior enhancement in the midline of neck, with homogenous internal echoes. Heterogeneous appearance may be seen due to the presence of echogenic fat, osseous, or dental elements. On CT, these lesions typically have simple fluid attenuation, with rim enhancement after intravenous contrast administration. Dermoid cysts have variable signal intensity on T1-weighted images. The epidermoid cysts may show increased T1 signal due to fat or protein component or isointense relative to muscle on T1-weighted images. The fat signal will be suppressed in STIR sequence. They are usually hyperintense on T2-weighted images. The lesion has a clearly demarcated rim but frequently has a heterogeneous internal appearance. MRI has got advantage of better soft tissue visualization, characterization, and multiplanar acquisition $[5,10,11]$. Epidermoid cysts always demonstrate a high signal intensity in MR DWI.

In large sizes USG, CT and MRI show this to be a plunging ranula, but histopathology confirms this to be epidermoid cyst; all the other possibilities of sublingual lesions rule out on the basis of histopathology and CT or MRI scans.

Differential diagnosis of cystic lesions of the floor of the mouth is important because the recommended surgical technique is not exactly the same in all of them. The differential diagnosis of submental and sublingual benign lesions are plunging ranula, thyroglossal duct cyst, cystic hygroma, mucocele, branchial cleft cyst, hemangioma, enlarged lymph nodes and infective lesions. For this reason, bimanual palpation and conventional radiography are not always sufficient in making differential diagnoses. In these cases, it is necessary to use USG, CT or MRI together with cytology examination by fine-needle aspiration (FNA) [1,12]. FNA is valuable diagnostic technique especially for excluding of the malignant cystic lesions [12].

Surgical enucleation is the only effective treatment for these kinds of lesions. The definite management of these lesions is complete surgical excision with very low recurrence rate, and it is facilitated by the fibrous capsule surrounding the cyst that makes it easy to be enucleated [13]. Several techniques are 
reported in the literature, which may be divided into intraoral and extraoral techniques depending on which approach is used. Extraoral approach is generally preferred in the case of median geniohyoid or very large sublingual cysts, whereas the intraoral approach is typically used for medium-sized sublingual cysts [14]. The intraoral approach is the most useful and effective for the treatment of large lesions and lead to very good cosmetic and functional results, whereas the extraoral incision was necessary only when the cysts were under the geniohyoid muscle [15]. There are no rules regarding the timing for operation; because epidermoid cysts are mainly congenital, they can appear in every age of life, so the time when they appear (generally with dysphagia, dysphonia, and dyspnea) is generally the right time to operate on them.

\section{Conclusion:}

When a cystic mass is detected on the floor of oral cavity, we must consider epidermoid cysts for differential diagnosis. USG, $\mathrm{CT}, \mathrm{MRI}$ and FNAC are essential for preoperative diagnosis and planning of surgical approach.

USG is frequently used firstly to confirm the cystic nature of the lesion. CT and MR imaging provide essential information on the cyst location and anatomical boundaries that allows optimal preoperative planning. USG evaluation sometimes can give all the necessary information before surgical intervention.

Thanks to the hyperintense signal showed by epidermoid cysts, DWI is very useful to identify and differentiate epidermoid cyst from other cystic lesions (in our cases from plunging ranula). DWI can also be useful for postoperative follow-up of epidermoid cysts, especially if not completely removed.

Complete surgical excision with intact capsule is the treatment of choice. If possible, intraoral approach should be preferred because of its perfect cosmetic results.

\section{References}

1. Vargas Fernandez JL, Lorenzo Rojas J, Aneiros Fernandez J, Sainz Quevedo M. Dermoid cyst of the floor of the mouth. Acta Otorrinolaringol Esp. 2007;58(1):31-33.

2. Kandogan T, Koc M, Vardar E, Selek E, Sezgin O. Sublingual epidermoid cyst: a case report. J Med Case Reports. 2007;1:87.

3. De Ponte FS, Brunelli A, Marchetti E, Bottini DJ. Sublingual epidermoid cyst. J Craniofac Surg. 2002;13(2):308-310.

4. Seah TE, Sufyan W, Singh B. Case report of a dermoid cyst at the floor of the mouth. Ann Acad Med Singapore. 2004;33(4):77-79.

5. Koca H, Seckin T, Sipahi A, Kaznac A. Epidermoid cyst in the floor of the mouth: report of a case. Quintessence Int. 2007;38(6):473-477.
6. Nissia Ananda, Arfan Badeges, Pradono. A Rare Case of Ranula on Ventral Surface of the Tongue. J Int Dent Med Res. 2016;9(Special Issue):408-412.

7. Kamil Serkan Agacayak, Ibrahim Kose, Nedim Gunes, Emrullah Bahsi, Ferhan Yaman, Serhat Atilgan. Dentigerous cyst with an impacted canine: case report. J Int Dent Med Res. 2011;4(1):21-24.

8. Eken M, Evren C, Sanli A, Bozkurt Z. An alternative surgical approach for sublingual dermoid cysts: a case report. Kulak Burun Bogaz Ihtis Derg. 2007;17(3):176-178.

9. Longo F, Maremonti P, Mangone G, Maria G, Califano L. Midline (dermoid) cysts of the floor of the mouth: report of 16 cases and review of surgical techniques. Plast Reconstr Surg. 2003;112(6):1560-1565.

10. Sandeep V, Jitendra KK, Sonka AA. Giant sublingual epidermoid cyst resembling plunging ranula. Natl J Maxillofac Surg. 2012;3(2):211-213.

11. Chikui T, Shimizu M, Goto TK, et al. Interpretation of the origin of a submandibular mass by CT and MRI imaging. Oral Surg Oral Med Oral Pathol Oral Radiol Endod. 2004;98(6):721-729.

12. Musayev J, Hasanov A, Aliyev B. An epidermal cyst in the submandibular gland: a case with fine needle aspiration image. Marmara Medical Journal. 2013;26:162-4.

13. El-Hakim IE, Alyamani A. Alternative surgical approaches for excision of dermoid cyst of the floor of mouth. Int J Oral Maxillofac Surg. 2008;37(5):497-499.

14. Modolo F, de Melo Alves S, Jr, de Almeida Milani B. Congenital teratoid cyst of the floor of the mouth. Otolaryngol Head Neck Surg. 2007;136:134.

15. Baliga M, Shenoy S, Poojary D. Epidermoid cyst of the floor of the mouth. Natl J Maxillofac Surg. 2014;5(1):79-83. 